\title{
Diagnostic Accuracy of High Resolution Ultrasonography in Comparison with MRI for Evaluation of Rotator Cuff Pathologies
}

\author{
Dineshram Vijayan ${ }^{1}$, Vinayagam Shanmugam ${ }^{2}$, Senthilkumar Aiyappan ${ }^{3}$, Premkumar Chidambaram ${ }^{4}$ \\ ${ }^{1}$ Junior Resident, Department of Radiodiagnosis, ${ }^{2}$ Professor and HOD, Department of Radiodiagnosis, ${ }^{3}$ Professor, \\ Department of Radiodiagnosis, ${ }^{4}$ Professor, Department of Radiodiagnosis, SRM Medical College Hospital, Kattankullathur, \\ Chennai-603203, India
}

Corresponding author: Dineshram Vijayan, 4/1102, Second Cross, Indira Nagar, Dharmapuri-636701, India

DOI: http://dx.doi.org/10.21276/ijcmsr.2020.5.2.3

How to cite this article: Dineshram Vijayan, Vinayagam Shanmugam, Senthilkumar Aiyappan, Premkumar Chidambaram.Diagnostic accuracy of high resolution ultrasonography in comparison with MRI for evaluation of rotator cuff pathologies. International Journal of Contemporary Medicine Surgery and Radiology. 2020;5(2):B8B12.

\section{A B S T R A C T}

Introduction: Rotator- cuff pathology is the most common cause of shoulder pain and disability. Rotator- cuff pathologies range from tendinosis to complete tears. The aim of this study was to evaluate the accuracy of Ultrasonography in the diagnosis of rotator cuff pathologies and to determine if Ultrasonography compares favourably in sensitivity and specificity to $\mathrm{MRI}$ in the diagnosis of rotator cuff pathologies.

Material and methods: 70 patients with suspected rotator cuff pathologies referred to radiology department for MRI examination were subjected to USG examination using a PHILIPS Affinity 30 Ultrasound machine and 1.5 Tesla SIEMENS MAGNETON ESSENZA MRI machine. The agreement between the two methods was assessed using kappa coefficient.

Results: Out of 70 patients, 67(95.7\%) were diagnosed to have rotator cuff pathologies, by using MRI as reference. The sensitivity, specificity, PPV and NPV of USG in detecting pathologies of Supraspinatus was $80.7 \%$,

$80 \%, 95.8 \%$ and $42.1 \%$, Infraspinatus was $45.5 \%, 96.4 \%, 71.4 \%$ and $90 \%$ and Subscapularis was $68 \%, 97.6 \%, 94.4 \%$ and $83.7 \%$ respectively. The agreement between USG and MRI was assessed using kappa coefficient, which showed the strength of agreement was "Good" between two modalities.

Conclusion: To conclude that, High resolution Ultrasonography is a cost effective, dynamic, easily available and acceptable Screening modality for rotator cuff pathologies in patients presenting with painful shoulder. There was a statistically significant agreement between Ultrasonography and Magnetic-resonance-imaging in diagnosis of 'Supraspinatus and Subscapularis' pathologies, but there was a 'weak' agreement between twomodalties in diagnosis of 'Infraspinatus' pathologies.

Keywords: MRI, USG, Rotator Cuff Pathologies

\section{INTRODUCTION}

Rotator- cuff pathology is the most common cause of shoulder pain and disability. ${ }^{1}$ Rotator- cuff pathologies range from tendinosis to complete tears and the prevalence ranges from $5 \%$ to $39 \%$, and rises with increasing age; it can increase markedly after 50 years of age and reach upto the peak of $80 \%$ in subjects over 80 years of age. ${ }^{2}$ Two noninvasive modalities, Ultrasonography (USG) and Magnetic Resonance Imaging (MRI) are widely used in evaluating rotator cuff pathologies. Low cost, wide availability and dynamic imaging are in favour of USG. Compared to USG, MRI is reported to have consistently high sensitivity (80 $-97 \%$ ) and specificity (93-94\%) for the diagnosis of rotator cuff pathologies. In this study, we estimated the diagnostic accuracy of USG in comparison with MRI. The purpose of this study is toevaluate the accuracy of Ultrasonography in the diagnosis of rotator cuff pathologies and to determine if Ultrasonography compares favourably in sensitivity and specificity to MRI in the diagnosis of rotator cuff pathologies.

\section{MATERIAL AND METHODS}

This was across-sectional study conducted in the department of Radiodiagnosis, SRM medical college hospital and research centre, Chennai from January 2018 to September 2019. 70 patients with suspected rotator cuff pathologies referred to radiology department for MRI examination were subjected to USG examination using a PHILIPS Affinity 30 Ultrasound machine with a $5-12 \mathrm{Mhz}$ broadband linear array transducer in both axial and longitudinal planes and then plain MRI was subsequently performed using 1.5 Tesla SIEMENS MAGNETON ESSENZA machine. The following MRI protocol [Table 1] was used with field of view for about $16-18 \mathrm{~cm}$ and slice-thickness for about $3 \mathrm{~mm}$ in our study.

Inclusion Criteria: All patients who were referred to the radiology department withClinically suspected rotator cuff 
injury and History of restricted movements in shoulder. Age group: 18-70 years.

Exclusion Criteria: Patients with previous history of any shoulder surgery, Shoulder implants, Cardiac pacemakers, Claustrophobia and Uncooperative during the study.

\section{Assessment}

Criteria used for rotator- cuff tear onusg in our study ${ }^{4}$

1. Complete Tears are Complete non-visualization of thecuff and localized non- visualization of tendon, establishedon minimum two planes.

2. Partial Tears can be further divided into tears of articularsurface, tears of bursal-surface and intra- substance tears. Features in USG are localised area of hypo- echogenicity or hetero- echogenicity spreading to either the articular surface or the tendon's bursal surface, Bursal-side flatenning of the rotator- cuff and Intra-substance tears were diagnosed by intra- tendinous fluid filled line not extending to the bursal or articular surfaces.

\section{Criteria of 'tendinosis' on USG ${ }^{4}$}

Tendinosis on USG appears as Hypoechoic tendons / thickened tendons and effacement of the fibrillar pattern.

\section{Criteria of rotator cuff tears on MRI'5}

1. Complete Tears are 'Hyperintense' defect on $\mathrm{T} 2$ weighted images or an avulsion of the tendon extending from the bursal surface to the articular surface of thecuff.
2. Partial Tears are a Focal area of 'hyperintensity' extending to only one surface, either the articular surface, the bursal surface, or within the tendon.

\section{Criteria of 'tendinosis'on MRI}

Tendinosis appears as Intrasubstance hyperintense area seen on PD- FS images which are ' not hyperintense' on T2 WI.

\section{STATISTICAL ANALYSIS}

The result analysis was done by using Statistical Package for the Social Sciences(SPSS) software, the various parameters like Sensitivity, Specificity, PPV and NPV were assessed and also the inter-modality agreement between Ultrasonography and Magnetic- resonance- imaging was assessed using Cohen's kappa-coefficient.

\section{RESULTS}

Our study group consists of 70 subjects from 16 to 72 years. The mean age of the population was 39.8 years. Majority of the subjects belongs to age less than 30 years. Most of them are males $50(71.4 \%)$ in our study group. Right side predominance of about $67.1 \%$ noted in our study group. Out of the 70 cases in the study group, 67 of them showed rotator cuff pathologies.

Supraspinatus was the most commonly affected tendon in our study, 61 out of 67 cases were diagnosed to have supraspinatus pathologies. Followed by Subscapularis tendon
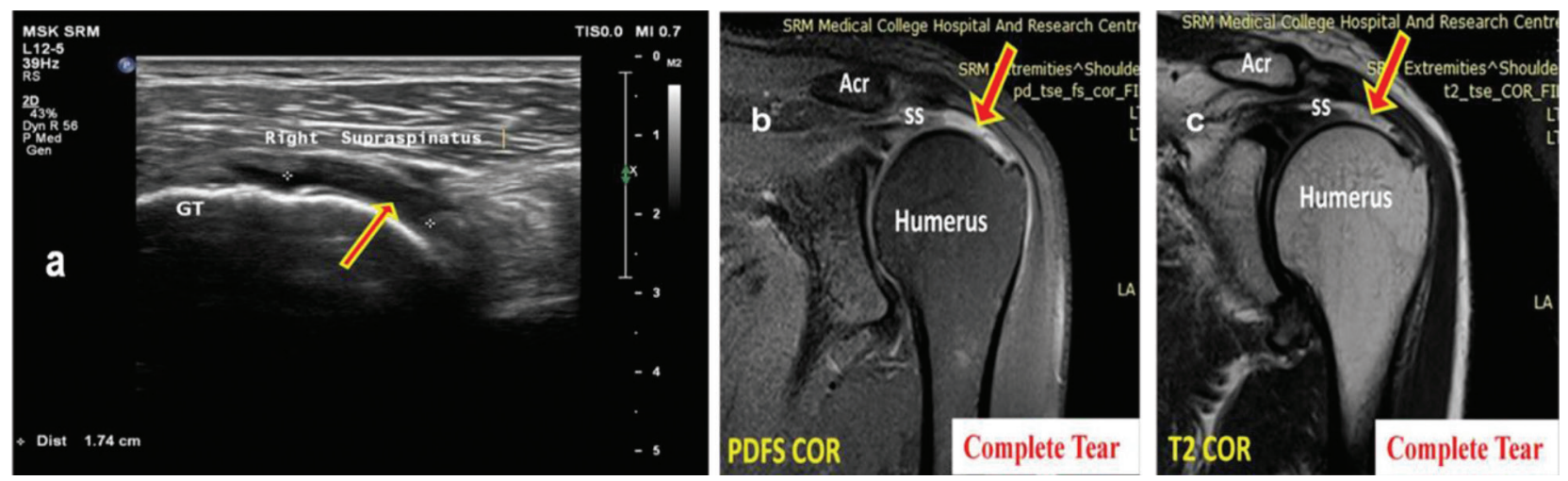

Figure-1: Supraspinatus complete tear. a) USG shows the focal non-visualization of supraspinatus tendon with retraction for $\sim 1.7 \mathrm{~cm}$.(Arrow). b) \& c) MRI of the same patient shows the hyperintense defect in T2WI coronal and PD- FS coronal sections respectively(Arrows).
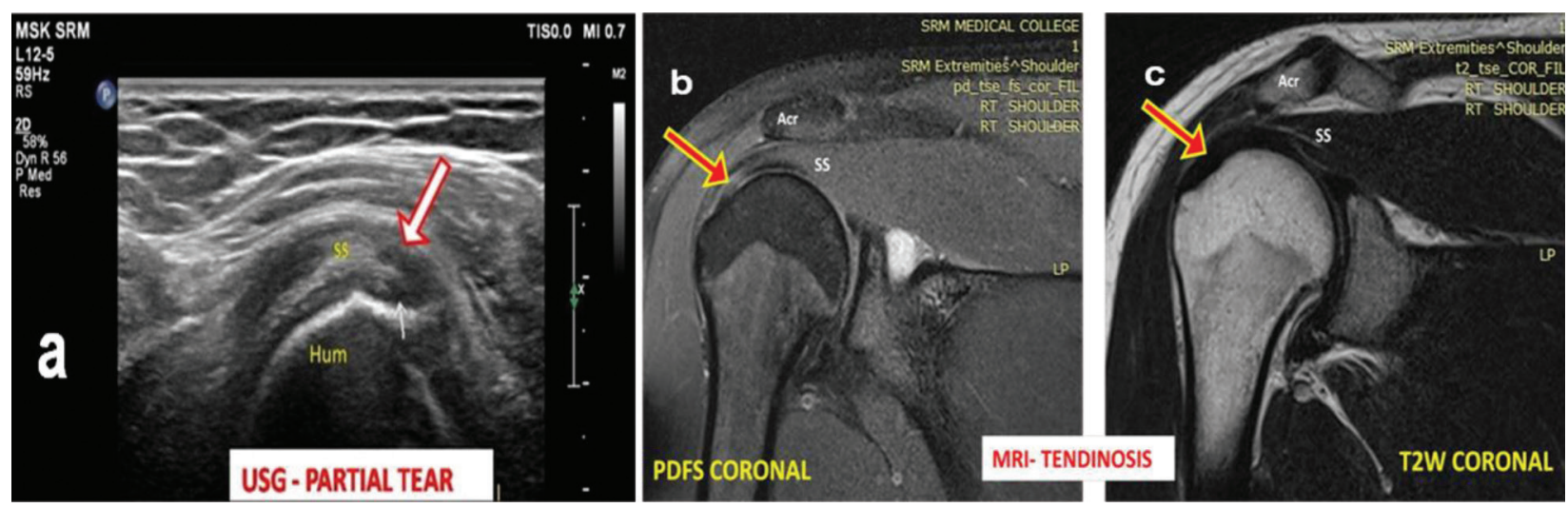

Figure-2: False positive supraspinatus partial tear. a) USG showsbursal surface 'partial tear' (Arrow) of supraspinatus (ss) tendon. b) MRI PD- FS coronal sequence of the same patient shows hyper-intense defect in SS tendon(Arrow).c) MRI T2WIofthe same patient shows normal intensity of SS tendon- Suggestive of 'Tendinosis'. 

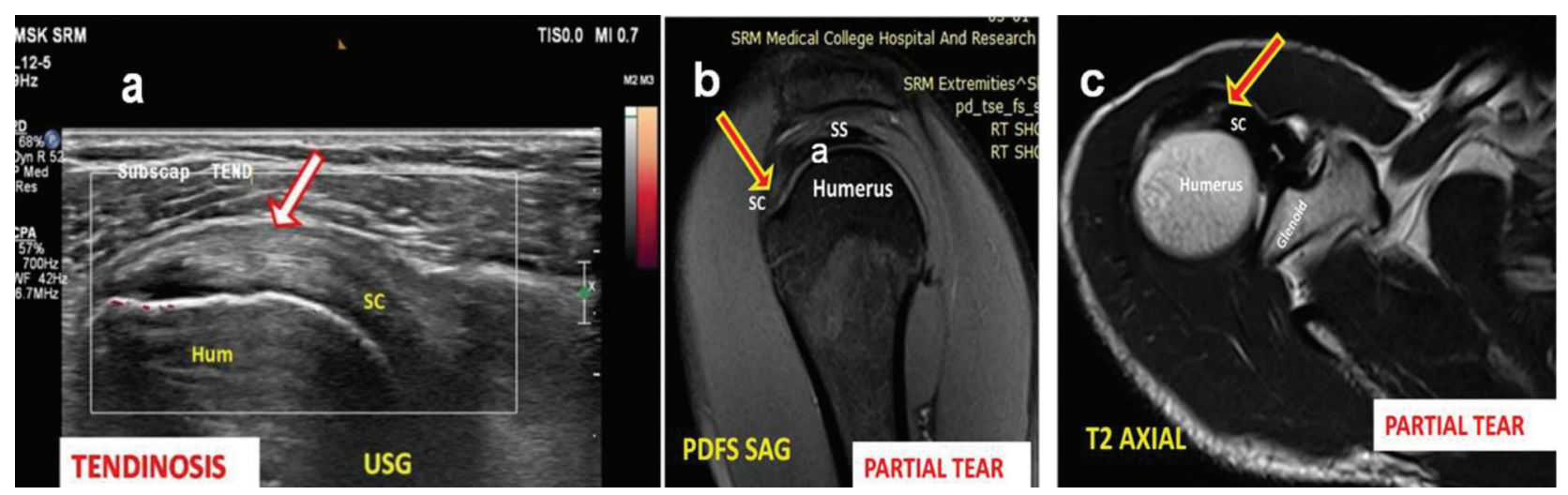

Figure-3: False negative subscapularis partial tear. a) USG shows'Tendinosis' of subscapularis(sc) tendon (Arrow). b) andc) MRI PD- FS SAGITTAL and T2WI- AXIAL of the same patient shows partial tear' of subscapularis(sc)tendon(Arrow).

\begin{tabular}{|l|l|l|}
\hline No & Orientation & Sequence type \\
\hline I. & Axial & $\begin{array}{l}\text { MEDIC/ GRE } \\
\text { PD- FS }\end{array}$ \\
\hline II. & Coronal & $\begin{array}{l}\text { PD- FS } \\
\text { T2 WI } \\
\text { T1 WI }\end{array}$ \\
\hline III. & & PD- FS \\
\hline \multicolumn{2}{|l|}{ Table-1: Protocol for MRI in evaluation of rotator-cuff injuries3 } \\
\hline
\end{tabular}

\begin{tabular}{|l|l|c|c|c|c|c|}
\hline \multicolumn{2}{|l|}{ Findings } & Senstivity (\%) & Specificity (\%) & PPV(\%) & NPV(\%) & KAPPA \\
\hline \multirow{3}{*}{ Complete tear } & Supraspinatus & 84.6 & 100 & 100 & 96.4 & 0.90 \\
\cline { 2 - 8 } & Infraspinatus & 60 & 100 & 100 & 96.9 & 0.74 \\
\cline { 2 - 8 } & Subscapularis & 66.7 & 100 & 100 & 98.5 & 0.79 \\
\hline \multirow{3}{*}{ Partial tear } & Supraspinatus & 76.9 & 90.7 & 66.7 & 94.2 & 0.6 \\
\cline { 2 - 8 } & Infraspinatus & 50 & 96.9 & 33.3 & 98.4 & 0.38 \\
\cline { 2 - 8 } & Subscapularis & 66.7 & 100 & 100 & 96.8 & 0.79 \\
\hline \multirow{3}{*}{ Tendinosis } & Supraspinatus & 72.4 & 97.4 & 95.5 & 92.2 & 0.7 \\
\cline { 2 - 8 } & Infraspinatus & 33.3 & 100 & 100 & 97.4 & 0.74 \\
\cline { 2 - 8 } & Subscapularis & 64.3 & 100 & 100 & 91.4 & \\
\hline
\end{tabular}

\begin{tabular}{|l|c|c|}
\hline Study & $\begin{array}{c}\text { Study } \\
\text { Population } \\
\text { (n) }\end{array}$ & $\begin{array}{c}\text { Kappa } \\
\text { Coefficient }\end{array}$ \\
\hline Shahnawaz et al, 2014 & 50 & 0.81 \\
\hline Chiristian Alexander et al, 2015 ${ }^{15}$ & 45 & 0.57 \\
\hline Amandeep et al, 2017 ${ }^{13}$ & 50 & 0.79 \\
\hline Abhinavpratap et al, 2017 & 60 & 0.63 \\
\hline OUR STUDY 2019 & 70 & 0.64 \\
\hline \multicolumn{2}{|c|}{ Table-3: Kappa coefficient values in different articles } \\
\hline
\end{tabular}

pathologies 32 out of 67 patients.

In our study, 'Sensitivity, 'Specificity, 'Positive Predictive Value(PPV) and 'Negative Predictive Value(NPV)' of USG in evaluating rotator cuff tendons 'partial tears' is $64.5 \%$, $95.8 \%, 66.6 \%$ and $96.4 \%$ respectively and for 'complete tears' is $70.4 \%, 100 \%, 100 \%$ and $97.2 \%$ respectively.

In our study USG of 'supraspinatus' tendon for detection of pathologies showed sensitivity of $80.7 \%$, specificity of $80 \%$, PPV of $95.8 \%$ and NPV of $42.1 \%$.No isolated case of an infraspinatus pathology noted in our study, there is always an associated supraspinatus pathology. USG of infraspinatus tendon pathologies in our study showed 'sensitivity' of 45.5\%, 'specificity' of 96.4\%, 'Positive Predictive Value(PPV) of $71.4 \%$, 'Negative predictive value(NPV) of $90 \%$.In our study Subscapularis is the second (53\%) most common tendon involved. Ultrasonography of subscapularis tendon pathologies showed the 'sensitivity of $68 \%$, "specificity of 97.6\%, 'Positive predictive value (PPV) of 94.4\%, ' Negative predictive value(NPV)' of $83.7 \%$. Teres minor appears normal in our study.

The comprehensive data of USG for evaluating both partial tears and complete tears, in terms of statistical analysis are presented in [Table 2/Figure 1,2,3]

The 'agreement' between the Ultrasonography and Magnetic resonance imaging for diagnosing rotator-cuff pathologies was determined using kappa- coefficient (Kappa $=0.64)$. The strength of 'agreement between Ultrasonography and Magnetic- resonance- imaging for the diagnosis of rotator- 
cuff tears is considered to be "Good".

\section{DISCUSSION}

'Rotator-cuff' pathologies are the 'most frequent' finding in patients with shoulder pain. In our study 67(95.7\%) out of 70 patients presented with rotator cuff pathologies and this correlates with the study carried out by Mitchel $\mathrm{C}$ et al in $2005 .^{6}$

In our study, the ages of all the cases ranged from 16 to 72 years (mean age 39.80 years). Out of these 70 patients; most of them $27(38.6 \%)$ patients were in age group less than 30 years. This finding is similar to the study done by Worland et al in $2003^{7}$ where most of the patients were less than 30 years age group. There was a male preponderance in our study, with $50(71.4 \%)$ subjects being males, which correlates with study conducted by Narvir et al $2016 .{ }^{8}$ There was rightshoulder dominance in our study with 47(67.1\%) being right dominance which is similar to the studies conducted by Bouazizet al 2010.9

Among the subjects studied, 'supra-spinatus' was the most commonly affected tendon in our study, 61 (91\%) out of 67 patients were diagnosed to have supraspinatus tendon pathologies, followed by subscapularis tendon pathologies $32(53 \%)$. In concordant withour study, study conducted by 'Iagnocco et al $2003^{10}$ have also established Supraspinatus to be the most frequently involved tendon.

In our study, 'Sensitivity, 'Specificity, 'Positive Predictive Value(PPV) and 'Negative Predictive Value(NPV)' of USG in evaluating rotator cuff tendons 'partial tears' is 64.5\%, $95.8 \%, 66.6 \%$ and $96.4 \%$ respectively and for 'complete tears' is $70.4 \%, 100 \%, 100 \%$ and $97.2 \%$ respectively. Our results showed 'better' specificity and PPV for 'partial' and ' complete' rotator- cuff tears when compared to the similar articles done by Vishal et al $2017^{4}$, Hiralet al $2017^{5}$ and Narvir et al $2016 .{ }^{8}$ Our study results showed 'lesser' sensitivity for ' partial' and ' complete' tears when compared to the similar studies done by Abhinav et al $2017^{11}$ and Saraya $S$ et al 2016. ${ }^{12}$

In our study USG of 'supraspinatus' tendon for detection of pathologies showed sensitivity of $80.7 \%$, specificity of $80 \%$, PPV of $95.8 \%$ and NPV of $42.1 \%$. Our results were comparable to the similar studies conducted by Abhinavetal $2017^{11}$ which showed the Sensitivity, Specificity, PPV and NPV of $78.7 \%, 84.6 \%, 94.8 \%$ and $52.3 \%$ respectively. Amandeep Singh et al, $2017^{13}$ showed the sensitivity and specificity of USG in evaluating 'supraspinatus'tendon are $89 \%$ and 43\%. Our results shows similar sensitivity and better specificitywhen compared to thisarticle.

Zehetgruber et $\mathrm{al}^{14}$ could not detectanyisolated infraspinatustears. Our study also had similar results. In allcases of an infraspinatus pathology, there was associated supraspinatus pathology also. USG of infraspinatustendon pathologiesin our studyshowed' sensitivity' of 45.5\%, 'specificity' of 96.4\%, 'Positive Predictive Value (PPV) of $71.4 \%$, 'Negativepredictivevalue (NPV) of $90 \%$. Our results were compared to the similar studies conducted by Abhinav et al $2017^{11}$ which showed the Sensitivity, Specificity, PPV and NPV of $45.5 \%, 96.4 \%, 71.4 \%$ and $90 \%$ respectively. Our resultsshows'less sensitivity' when compared to Abhinav et al $2017^{11}$ due to less number of subjects with infraspinatuspathology.

In our study 'Subscapularis' is the second (53\%) most common tendon involved. Ultrasonography of subscapularistendon pathologiesshowed the 'sensitivity of 68\%, 'specificity of $97.6 \%$, 'Positive predictive value (PPV) of 94.4\%, ' Negative predictive value (NPV)' of $83.7 \%$. Our results were 'comparable' to the similar studies conducted by Abhinav et al $2017^{11}$ which showed the Sensitivity, Specificity, PPV and NPVof 83.3\%, 96. 2\%, 71.4\% and 98. 1\% respectively. Amandeep Singh et al, $2017^{13}$ showed the sensitivity of USG in evaluating subscapularis tendon is 30\%. Our results shows 'less sensitivity' when compared to Abhinav et al $2017^{11}$ study and 'better sensitivity' when compared to Amandeep Singh et al, $2017^{13}$ study, which showed sensitivity and specificity of $39.5 \%$ and $93.1 \%$ of USG in diagnosing Subscapularis pathologies.

We could demonstrate 'no' pathologies in teres-minor tendon out of 70 subjects, which was similar to the study conducted by Abhinavpratap et al $2017^{11}$, where out of 60 subjects all subjects have normal teres minor tendon. In contradictory to our findings Narvirsingh chauhan et al $2016^{8}$ demonstrated 1 case of partial tear (3.2\%) of teres- minor tendon on both USG and MRI, However teres-minor is the 'least' commonly affected tendon in their study.

The ' agreement' between the Ultrasonography and Magnetic resonance imaging for diagnosing rotator-cuff pathologies was determined using kappa- coefficient (Kappa=0.64). The strength of 'agreement between Ultrasonography and Magnetic- resonance- imaging for the diagnosis of rotatorcuff tears is considered to be "Good". Our results were nearly 'comparable' with the similar studies done by Shahnawaz et al $2014^{1}$, Abhinav pratap et al $2017^{11}$ and Amandeep et al 2017. ${ }^{13}$

Chiristian Alexander et al $2015^{15}$ showed "Moderate" agreement between two modalities in diagnosing rotator cuff pathologies. In condractictory to this study our study showed "Good" agreement between two modalities in diagnosing otator cuff pathologies.[Table 3]

Our study proves that Ultrasound is accurate and reliable in diagnosing a wide range of shoulder disorders compared with MRI. Similar to our study, Kenn W et al $2000^{16}$ studyhave found that Ultrasound is accurate and reliable in diagnosing a wide range of shoulder disorders when compared with MRI.

\section{Limitations}

Despite being the gold standard for diagnosing rotator- cuff tears, due to its invasive nature arthrography was not included in this study and plain magnetic-resonance- imaging, which is known to be a highly accurate in diagnosing rotator- cuff tears, has been taken as the gold-standard instead.

Small number of subjects with Infraspinatus pathology was found in our study. So, further studies with more significant number of infraspinatus pathologies are suggested to overcome these factors.

\section{CONCLUSION}

In our study, Supraspinatus is the most frequently involved tendon in rotator- cuff pathologies. There was a statistically significant agreement between Ultrasonography and 
Magnetic- resonance-imaging in diagnosis of 'Supraspinatus and Subscapularis' pathologies, but there was a ' weak' agreement between the two modalties in diagnosis of " Infraspinatus' pathologies. To conclude that, High resolution Ultrasonography is a cost effective, dynamic, easily available and acceptable Screening modality for rotator- cuff pathologies in patients presenting with painful shoulder.

\section{REFERENCES}

1. ShahnawazBashir, Syed RuzinaFirdose, Youniskamal, Hayat Ahmad khan et al, Correlation between High Resolution Ultrasonography and MRI in RotatorCuff TearDiagnosis, International Journal of Health Sciences \&Research. 2014;4(8):104-12.

2. MilgromC, SchafflerM, Gilbert S et al: Rotator cuff changesin asymptomatic adults, the effect of age, hand dominance and gender. J Bone joint surg Br. 1995; 77(2):296-8.

3. William C. McMaster, Andrew Roberts and Terry Stoddard; A correlation betweenshoulder laxity and interfering pain in competitive swimmers. Am J Sports Medial. 1998;26(1): 83-86.

4. Vishal DhirenbhaiThakker, DipuBhuyan, ManaliArora and MohsinaIslam Bora, Rotator Cuff Injuries:Is Ultrasound Enough?A Correlation with MRI, International Journal ofAnatomy, Radiology and Surgery. 2017;6(3):RO01-RO07.

5. HiralHapani, MahakSood, AnjanaTrivedi, Anirudh Chawla et al: Ultrasound, MRI and arthroscopic correlation of rotator cuff tears. International Journal of Contemporary Medical Research 2017;4(3):650-652.

6. Mitchell C, AdebajoA, Hay E, Carr A. Shoulder pain: diagnosis and management in primary care. BMJ. 2005;331(7525):1124-28.

7. Worland RL, Lee D, Orozco CG, SozaRex F, Keenan J. Correlation of age, acromial morphology, and rotator cuff tear pathology diagnosed by ultrasound in asymptomatic patients. J South Orthop Assoc. 2003;12(1): 23-6.

8. Narvir Singh Chauhan, Ajay Ahluwalla, Yash Paul Sharma and LokeshThakul. A Prospective Comparative Study of High Resolution Ultrasound and MRI in the Diagnosis of Rotator Cuff Tears in a Tertiary Hospital of North India, Pol J Radiol, 2016; 81(3):491-497.

9. Bouaziz MC, Jabnoun F, Chaabane S, Ladeb MF. Diagnostic Accuracy of High Resolution Ultrasound in Communicating Rotator Cuff Tears. Iran J Radiol. 2010;7(3):153-160.

10. Iagnocco A, Coari G, Leone A, ValesiniG. Sonographicstudy ofpainful shoulder. Clinical and Experimental Rheumatology. 2003;21(3):355-58.

11. Abhinavpratapsingh, Anuradha Rao, Siddalinga Devaruand Amithavikrama, Role of Ultrasound in Evaluation of Shoulder Injuries: A Comparative Studyof Ultrasound and MRI, International Journal ofAnatomy, Radiology and Surgery. 2017; 6(1):RO12-RO18.

12. Saraya S, El Bakry R, Ultrasound: Can it replace MRI in the evaluation of the rotatorcuff tears? Egyptian JournalofRadiology and Nuclear medicine. 2016;47(1):193-201.

13. Amandeep Singh, ChuniLalThukral, Kamlesh Gupta,
Mahesh Inder Singh et al, Role and Correlation of High Resolution Ultrasound and Magnetic Resonance Imaging in Evaluation of Patientswith Shoulder Pain, PolJRadiol. 2017;82:410-417.

14. Zehetgruber H, Lang T, Wurnig C. Distinction between supraspinatus, infraspinatusand subscapularistendon tears with ultrasound in 332 surgically confirmed cases. Ultrasound Med Biol. 2002; 28(6): 711-17.

15. Christian Alexander Fischer, Weber MA, NeubeckerC, Bruckner Tetal. Ultrasound vs. MRI in the assessment of rotatorcuff structure prior to shoulder arthroplasty; journal of orthopaedics. 2015;12(1):23-30.

16. KennW, HufnagelP, Muller T, GohlkeF, BohmD. Arthrography, ultrasound and MRI in rotator cuff lesions: a comparison of methods in partial lesions and small complete ruptures. Rofo. 2000; 172(3):260- 6.

Source of Support: Nil; Conflict of Interest: None

Submitted: 24-03-2020; Accepted: 13-04-2020; Published online: 10-05-2020 